\title{
MICRO-OPTICS
}

\section{Light sprinkler}

Although high rotation rates have been achieved with optically driven microrotors, particularly in low-viscosity fluids or with small rotor sizes to minimize drag, the demonstrated torque efficiency is typically small in practice compared with theoretical maxima. Now, Silvio Bianchi and colleagues in Italy and Hungary have demonstrated that with precise rerouting of light in 3D structures fabricated by direct laser writing, optical micro-turbines can better harness light's momentum and efficiently generate uniform and controllable torque (Nat. Commun. 9, 4476; 2018).

The corresponding author, Roberto Di Leonardo, told Nature Photonics that the motivation for the work originated from the simple realization that previous designs for light-driven micro-rotors actually generate a torque, per unit optical power, that is well below the theoretical maximum. To achieve greater efficiency the team came up with a complex $3 \mathrm{D}$ microstructure to reroute incoming light towards multiple output points in a way that maximizes mechanical torque. The structure, made of SU8 photoresist with a chiral shape, looks like the type of garden sprinkler for which water is guided out through multiple arms to eject water, causing the structure to rotate. In their work, the watercarrying sprinkler arms are substituted by microfibre optical waveguiding structures designed to eject light from the radial periphery of the structure (see image).

While most optical turbines depend on scattering, here it is waveguiding that enables much of the incident light to reach the desired outlet points from

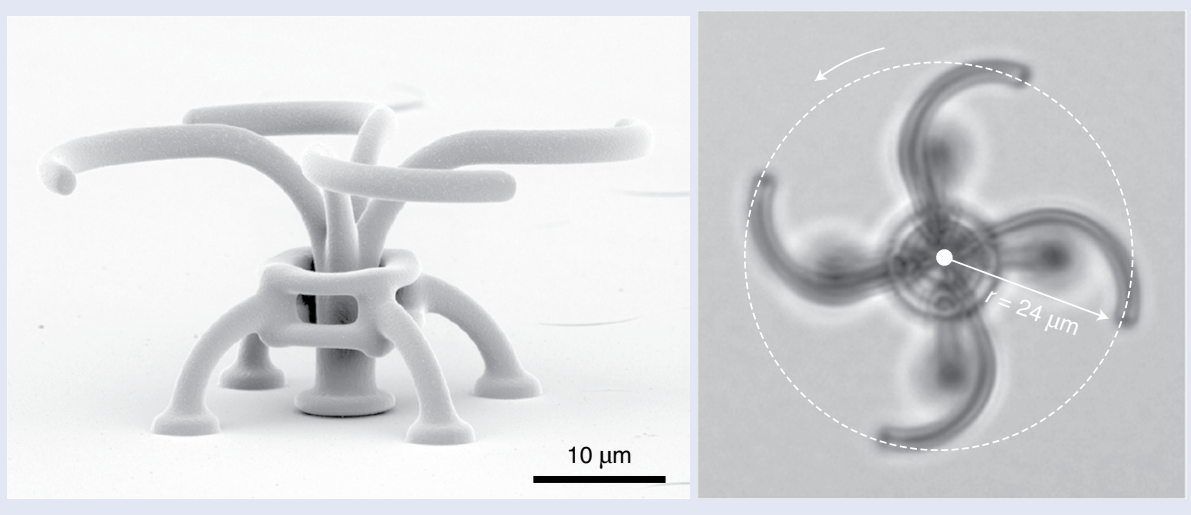

Credit: reproduced under a Creative Commons licence (https://creativecommons.org/licenses/by/4.0/)

which light escapes. This design maximizes the practically achievable reaction torque and the authors say it can be of the same order of magnitude of the ideal maximum value.

Di Leonardo explained that to fabricate the geometry of the garden sprinkler they used a home-built direct laser writing set-up based on two-photon polymerization. While the 3D fabrication is impressive, Di Leonardo mentioned that the most challenging aspect of the work was actually dealing with guiding light through the complex and sharply bent sections (curvature radii $\sim 10 \mu \mathrm{m}$ ). It was necessary to understand the ramifications of the bends and quantitative analysis was conducted for the losses when light propagates inside complex networks of optical fibres. The analysis of curved microfibres led to the particular shape parameters of the structure.
"Optical tweezers are often used to trap and manipulate simple spherical objects. We demonstrated that light actuation can be more efficient when using structures that are capable of guiding light in a controlled and efficient way," Di Leonardo said. "Using modern 3D microfabrication techniques will allow design of complex objects that will make optical micromanipulation more efficient and versatile."

The team is currently working on using shaped laser beams to improve coupling into the structures, and also on the simultaneous control of arrays of rotors inside microfluidic chips for the controlled generation of microflows.

\section{David F. P. Pile}

Published online: 28 November 2018 https://doi.org/10.1038/s41566-018-0316-z 